\title{
Vida y materia: Bergson y la Termodinámica clásica
}

\author{
RONALD DURÁN ALLIMANT \\ Universidad de Playa Ancha (Chile) \\ ronald.duran@upla.cl
}

\begin{abstract}
Resumen
La termodinámica clásica establece una oposición irreducible entre vida y materia. El universo dibujado por ella tiende de manera irreversible, debido a una continua disipación de energía, a un estado de equilibrio o reposo, la llamada «muerte térmica». En este universo, la vida, su actividad y su evolución aparecen como externas y fortuitas, permitidas, pero no explicadas por las leyes de la termodinámica. La vida aparece casi anti-natural o milagrosa en un universo muerto o en vías de morir. El filósofo francés Henri Bergson intenta superar esta contraposición de vida y materia con su concepción de un universo en continua creación conducido por un élan vital. Este impulso vital es fundamento tanto de la vida como de la materia, y la organización de los seres vivos y su evolución, la evolución creadora, necesitan de ambos principios para su concreción. Sin embargo, la propuesta de Bergson no logra establecer una verdadera unidad de vida y materia. Mantiene la dualidad de estos principios, a pesar de su complemento, y deja sin responder por ejemplo cómo es posible el surgimiento de la vida a partir de la propia materia.
\end{abstract}

Palabras clave: vida, materia, disipación, termodinámica, evolución, Bergson.

\section{Life and matter: \\ Bergson and the classical Thermodynamics}

\begin{abstract}
Classical thermodynamics establishes an irreducible opposition between life and matter. The universe draws by classical thermodynamics tends to, due to a continuous dissipation of energy, an equilibrium or state of rest, the so-called «heat death». In this universe, life, her activity and her evolution, appear as external and fortuitous, permitted but not explicated by laws of thermodynamics. Life seems unnatural or miraculous in a dead or almost dead universe. The French philosopher Henri Bergson tries to overcome this opposition between life and matter with bis conception of a universe in an unending creation, driven by an élan vital, which is the foundation of both life and matter. Organization of living beings and their evolution, the creative evolution, need both principles for its concretion. However, the Bergson's proposal fails to make a real unity of life and matter, keeping the duality of these principles, even when he conceives them as a complementary pair, leaving unanswered for example how it is possible the emergence of life from matter itself.
\end{abstract}

Key words: life, matter, dissipation, thermodynamics, evolution, Bergson.

Doctor en Filosofía por la Pontificia Universidad Católica de Valparaíso, Chile. Actualmente se desempeña como profesor en el Departamento de Filosofía de la Universidad de Playa Ancha (UPLA), y como investigador postdoctoral en el Instituto de Filosofía de la PUCV, financiado por un proyecto interno DI de dicha universidad.

Recibido: 7/Agosto/2015 - Aceptado: 30/Septiembre/2015 


\section{Introducción: la oposición de vida y materia}

La relación de vida y materia, y la comprensión de los seres vivos a partir de las leyes de la física ha sido siempre problemática. Así lo testimonian las distintas disputas de vitalistas y mecanicistas a lo largo de la historia.

De acuerdo a la teoría mecanicista, los fenómenos biológicos son sólo complicadas relaciones de procesos físicos y químicos [...] La teoría vitalista [por su parte] niega la posibilidad de la reducción de los procesos vitales a procesos físicos y químicos. Se necesitan principios "especiales", diferentes de los procesos físicos y químicos, para la "actividad" de los organismos vivos (Bertalanffy, 1933: 28).

El carácter especial de los seres vivos se destaca una y otra vez en cada nuevo intento de reducción a meros procesos físico-químicos, aunque las teorías y herramientas de la física se amplían y desarrollan volviendo más plausible una comprensión física de lo biológico. Dentro de esta problemática general, las relaciones entre evolución biológica y leyes de la termodinámica ocupan un lugar especial. Las concepciones de naturaleza y del universo elaboradas por ellas durante el siglo XIX, pusieron en oposición de manera radical la vida y la materia. La segunda ley de la termodinámica, asociada a los procesos disipativos, sin prohibir la vida, la vuelve improbable, excepcional, y extrínseca. La materia y la vida aparecen como contradictorias en el marco de la termodinámica clásica. «Desde el siglo XIX, el problema de la oposición entre las leyes de la materia sometida al segundo principio y las leyes de la evolución biológica está puesto claramente y veremos manifestarse numerosas tentativas de conciliación entre estas dos orientaciones aparentemente irreducibles» (Prigogine \& Glansdorff, 1973: 675). Se plantea así una oposición entre la dirección de la «evolución» termodinámica y la de la evolución biológica, entre la tendencia al reposo y la destrucción de estructuras, y la continua creación de formas nuevas y cada vez más complejas, tanto biológicas como sociales.

El desarrollo de la ciencia moderna ha conducido a una curiosa divergencia de visiones de mundo. Para los astrónomos y los físicos, el mundo está, por así decir, "cayendo" hacia un estado de inercia mortal en el que el calor está uniformemente distribuido a través de él. Para los biólogos y sociólogos, una parte del mundo (y para nosotros una parte muy importante), experimenta, de todas maneras, un progresivo desarrollo en el que aparece una tendencia hacia arriba, donde estados de organización más bajos son reemplazados por estados más altos (Needham, 1942: 352). 
Se plantea así un conflicto aparentemente irreducible entre vida y materia.

En este artículo estudiaremos la propuesta de conciliación de este conflicto ofrecida por el filósofo francés Henri Bergson (1859-1941), en su obra L'évolution créatrice [La evolución creadora] (1907). Para ello seguiremos los siguientes pasos: presentaremos, en primer lugar, la imagen de universo de la termodinámica clásica y la oposición vida y materia que se da en ella; en segundo lugar, mostraremos la imagen de universo de Bergson y su intento de conciliación de vida y materia, al incorporar las leyes de la termodinámica en un universo en continua creación, conducido por un élan vital; en tercer lugar, veremos cómo conjuga Bergson vida y materia en la organización de los seres vivos y en la evolución biológica, la evolución creadora; por último, mostraremos la insuficiencia de esta propuesta de superación de la oposición vida y materia, al mantener un dualismo que no logra configurar una verdadera unidad.

\section{Termodinámica clásica: la vida en un universo que muere}

En la termodinámica clásica, la vida parece proscrita por la segunda ley. Ésta implica que los procesos disipativos conducen irreversiblemente el universo a un estado final de equilibrio o reposo, la llamada «muerte térmica» (Kragh, 2008), siguiendo un curso de creciente inactividad y destrucción de estructuras. La vida y su evolución resultarían meramente provisorias, excepcionales e improbables. La imagen aquí esbozada, donde la actividad de la naturaleza tiende a su propia anulación en el reposo, contrasta con la actividad creciente y abierta de la evolución biológica (Glansdorff \& Prigogine, 1971: 287).

El primer paso en la configuración de esta imagen se debe al ingeniero francés Sadi Carnot (1796-1832) quien en sus Reflexiones sobre la potencia motriz del fuego de 1824, ofrece la primera formulación de la segunda ley, afirmando que toda producción de trabajo mecánico en una máquina de vapor se debe a una diferencia de temperatura, es decir, a una conducción de calor que tiende a homogeneizar las temperaturas (Carnot, 1987: 41). Para obtener trabajo no basta tener un cuerpo caliente, es necesario un paso de calor de un cuerpo más caliente a uno más frío. Desde la perspectiva ingenieril, esto implica que la eficiencia de las máquinas nunca es perfecta, su funcionamiento conlleva siempre conducción de calor y por tanto una pérdida. Sin embargo, esta pérdida, esta conducción de calor, es justamente lo que posibilita la actividad o el movimiento en que consiste el trabajo mecánico. Sin ella, no habría proceso de conversión o transformación de energía. 
Las ideas de Carnot serán sistematizadas y generalizadas, más allá de las cuestiones tecnológicas, por Thomson y Clausius (Prigogine \& Stengers, 1994: 158). El físico británico William Thomson (Lord Kelvin) (1824-1907), afirma en 1852 que hay una "tendencia universal a la disipación de la energía mecánica" (Thomson, 1882), introduciendo con esto el término "disipación" para referirse a los procesos irreversibles. Las conversiones de energía son siempre imperfectas en los procesos reales, siempre hay energía potencial no aprovechada como trabajo, siempre hay energía disipada, que se pierde como movimiento y agitación molecular (Thomson, 1892). El término "disipación" tiene aparejadas connotaciones morales negativas, sobre todo en la sociedad victoriana a la que pertenecía Thomson, tan partidaria de la austeridad y el ahorro. "El término 'disipación' es sinónimo de 'intemperado, disoluto, o modo de vida vicioso...despilfarro, gasto...' en la cultura de la industrializada Inglaterra victoriana" (Brush, 1976: 551). Aquello que permite la actividad, el propio proceso disipativo, es mentado aquí sólo como pérdida o disipación. Que todo proceso implique irreversibilidad, por tanto una irrecuperabilidad, una modificación real, aparece como un defecto desde el punto de vista de la conservación y la equivalencia. La propia idea de "disipación de energía" privilegia este punto de vista, puesto que considera la energía como separada del proceso que la manifiesta. Se considera la energía desde una perspectiva estática, como energía potencial almacenada o "stored", como dice Thomson (1882: 513), energía disponible e indestructible.

La segunda gran ley de la Termodinámica involucra un cierto principio de acción irreversible en la naturaleza [...] a pesar de que la energía mecánica es indestructible, existe una tendencia universal a su disipación, que produce un aumento y difusión gradual del calor, cese del movimiento, un agotamiento de la energía potencial a través del universo material. El resultado sería inevitablemente un estado de reposo y muerte universal (Thomson, 1889: 349).

En este universo imperfecto de disipación y pérdidas, este universo que muere, que se agota a cada instante por su propia actividad, la vida es meramente pasajera, tiene lugar en un espacio de tiempo acotado tanto hacia el pasado como hacia el futuro (Thomson, 1882: 514; 1889: 349). La disipación vuelve provisoria la vida, destino que ni la propia actividad vital puede revertir.

Dentro de un periodo finito de tiempo pasado, y dentro de un periodo de tiempo por venir, la tierra debe haber sido y volverá a ser, no apta para la 
ocupación del hombre tal como está constituido al presente, a menos que se hayan realizado o sean realizadas, operaciones que son imposibles bajo las leyes a las que están sujetas las operaciones que se llevan a cabo actualmente en el mundo material (Thomson, 1882: 514).

Thomson intenta superar esta visión trágica del universo apelando al carácter infinito de la materia:

Es imposible concebir un límite a la extensión de materia en el universo; y por lo tanto, la ciencia apunta más bien a un progreso sin fin, a través de un espacio sin fin, de la acción que implica la transformación de energía potencial en movimiento palpable y de aquí en calor, más que a un mecanismo finito, agotándose como un reloj, y deteniéndose para siempre (Thomson, 1889: 349).

El físico alemán, Rudolf Clausius (1822-1888), por su parte, expresa cosmológicamente las leyes de la termodinámica en 1865: "La energía del universo es constante. La entropía del universo tiende a un máximo [Die Energie der Welt ist constant. Die Entropie der Welt strebt einem Maximum zu]" (Clausius, 1867: 365). La función entropía, denominada así por Clausius para asemejarla a la energía y que significa transformación (Clausius, 1867: 357), cuantifica los procesos irreversibles, el desbalance entre procesos disipativos y procesos reversibles o conservativos. El universo, sistema aislado de energía constante, tiende a un estado de máxima entropía, donde toda su energía disponible se "pierde" en calor. "En cuanto más el universo se aproxima a esta condición límite en la cual la entropía es un máximo, más disminuyen las ocasiones de cambios posteriores; y suponiendo que esta condición sea al fin alcanzada, ningún cambio podría tener lugar nunca más, y el universo estaría en un estado de muerte inmutable" (Clausius, 1868: 419). Este estado final es el estado de equilibrio o muerte térmica, donde desaparecen todas las diferencias de temperatura y ya no hay ningún tipo de actividad macroscópica. El equilibrio es sinónimo de reposo y muerte.

El estado de equilibrio y el camino irreversible hacia él, adquieren un nuevo sentido en la obra del físico austríaco Ludwig Boltzmann (18441906). En el marco de la concepción atomística y mecánica de la naturaleza, Boltzmann plantea una interpretación puramente probabilista de la entropía (Boltzmann, 1972; 1964). El camino irreversible hacia el equilibrio implica el paso de un estado menos probable a otro más probable, que en sentido físico implica mayor desorden molecular (Boltzmann, 1986: 67). El equilibrio es el estado más probable y el más desordenado. Así, disipación es sinónimo además de probabilidad y desorden. Boltz- 
mann esboza una imagen trágica del universo, que como un todo está desde siempre en equilibrio, un universo muerto, en el que se producen fluctuaciones o desvíos del equilibrio que corresponden a mundos como nuestro sistema solar, en los cuales se dan procesos irreversibles regidos por la segunda ley. Estos mundos de no-equilibrio, estas fluctuaciones, tarde o temprano se atenúan (Boltzmann, 1964: 447; 1895: 415). La segunda ley adquiere así un sentido meramente local, válida sólo para los mundos particulares y no para el universo en su conjunto. La vida sólo es posible en estos mundos, es una isla en medio de la muerte universal.

En el universo, que está como un todo en equilibrio térmico y por tanto muerto, ocurrirá que por aquí y por allá, pequeñas regiones, del mismo tamaño de nuestra galaxia (que llamaremos mundos), fluctúan considerablemente a partir del equilibrio térmico [...] estas regiones pequeñas y aisladas, se hallarán siempre "inicialmente" en un estado improbable. Este método me parece la única manera en la que podemos entender la segunda ley - la muerte térmica de cada mundo en particular - sin un cambio unidireccional del universo entero desde un estado inicial a un estado final (Boltzmann, 1964: 446).

Insignificante y provisoria, convertida en una mera fluctuación improbable, la vida lucharía por aprovechar la energía disponible que se agota a cada instante. Lucha a contracorriente que tiene un fin inevitable en la muerte térmica.

La lucha general por la existencia de los seres vivos no es una lucha por los elementos básicos -los elementos básicos de todos los organismos existen en abundancia en el aire, en el agua y el suelo-, ni tampoco por la energía [disipada] que se encuentra contenida en forma de calor, desgraciadamente inutilizable, en cada cuerpo, sino una lucha por la entropía, que sólo se encuentra disponible en la transmisión de la energía del sol caliente a la tierra fría (Boltzmann, 1986: 72).

La entropía indica procesos disipativos y por tanto energía disponible, que es aprovechada por las plantas a través de la fotosíntesis. La disipación permite la vida, pero sólo de manera provisoria y extrínseca. La vida no nace de la materia, sino que se sostiene apenas siguiendo el curso inverso de esta. La constante actividad vital no se sigue de las leyes de la termodinámica, sino que aparece para éstas como improbable y extraña. En el universo en disipación de la termodinámica clásica, la vida y su evolución sólo tienen lugar como una excepción provisoria. La actividad vital se hunde en la tendencia a la inactividad del mundo material. En esta imagen de la naturaleza, la materia es entendida como puramente 
disipativa, en un sentido negativo, como puramente destructiva de las formas, estructuras y ordenaciones que están inscritas en ella, pero que no han nacido de ella. Es así que la vida y su actividad creativa, parecen no tener lugar, parecen precarias y provisorias, sosteniéndose en contra y a pesar de la irreversibilidad, de una manera casi anti-natural y milagrosa. Se establece así una radical brecha entre vida y materia, entre creación y disipación. En el universo muerto o en vías de morir de la termodinámica clásica, la vida aparece sólo como un desvío, como una fluctuación pronta a desaparecer, como una actividad extraña y ajena a la materia. La imagen de universo que dibujará Bergson será justamente la opuesta. En ella la vida será el elemento fundamental.

\section{Bergson: vida y materia en un universo en creación continua}

El filósofo francés Henri Bergson, parte de una posición diametralmente opuesta a la de la termodinámica clásica. Su modelo no son las máquinas de vapor y su actividad decreciente, sino la actividad constante y creciente de los seres vivos y su evolución. La actividad vital, con su incesante creación de formas, la irreversibilidad e imprevisibilidad de la vida, el impulso vital que parece recorrer a cada uno de los seres vivos, constituyen la fuente de inspiración de su concepción e imagen de naturaleza. La vida no es ya excepcional en un universo muerto o en vías de morir sino su carácter esencial y más propio.

El universo, en Bergson, es vida, duración, irreversibilidad, continua creación (Bergson, 2007: 30, 342, 277). "La naturaleza se nos aparece como una inmensa eflorescencia de imprevisible novedad" (Bergson, 1963: 857). Este universo, esta naturaleza, son evolución creadora. Un impulso o corriente vital (élan vital) recorre y constituye el universo, y es fuente última tanto de la vida como de la materia (Bergson, 2007: 30). Impulso que, caracterizado como libertad (Bergson, 2007: 269), querer (Bergson, 2007: 245), conciencia (Bergson, 2007: 266), energía espiritual (Bergson, 1963), devenir y flujo continuo (Bergson, 2007: 255), es "exigencia de creación" (Bergson, 2007: 257). Cada una de las formas de vida particular y su evolución siguen este impulso, van en su misma dirección, sin embargo, el impulso vital no se agota en ninguna de ellas, sino que las trasciende. "Como remolinos de polvo levantados por el viento que pasa, los vivientes giran sobre sí mismos, suspendidos por el gran soplo de la vida" (Bergson, 2007: 141). Al impulso se le opone, resistiéndole, un movimiento contrario, cuyo carácter dinámico depende, sin embargo, del propio impulso vital: la materia (Bergson, 2007: 365). Materia que es movimiento o tendencia hacia la espacialidad, donde reina el automatismo, el determinismo y la necesidad, al menos como tendencias (Bergson, 
2007: 30). Bergson ofrece distintas imágenes o metáforas para expresar su concepción de la naturaleza, para expresar el impulso vital y su relación con la materia y los seres vivos: un chorro de vapor (Bergson, 2007: 253), el movimiento libre de un brazo (Bergson, 2007: 254), fuegos artificiales (Bergson, 2007: 257), mano que empuja limaduras de hierro (Bergson, 2007: 109), onda que se propaga (Bergson, 2007: 271), ola (Bergson, 2007: 273), y corriente (Bergson, 2007: 270), entre otras.

Como hemos dicho, un mismo impulso es fuente de una doble dirección. Sin el doble movimiento de vida y materia, no habría creación ni concreción alguna. No habría mundos, ni organismos, ni evolución. Sin materia el élan vital se mantendría en una confusión indiferente de virtualidades sin cuerpo (Bergson, 2007: 263). Sólo hay creación cuando hay conjunción de vida y materia, de necesidad y libertad. "La materia es primero lo que divide y lo que precisa [...] La materia distingue, separa, resuelve en individualidades y finalmente en personalidades, tendencias en otro tiempo confundidas en el impulso original de la vida" (Bergson, 1963: 855). La materia viene a dar cuerpo a lo virtual, es principio de individuación y concreción de las virtualidades del impulso vital. En sentido propio, sólo hay esfuerzo o impulso, porque hay oposición, resistencia. Esfuerzo que permite que se saque más de lo que se tenía, "más" que indica la novedad absoluta, la creación (Bergson, 1963: 856). ¿En qué consistiría un impulso sin oposición? Sería pura interioridad ya cumplida, no proceso ni creación, sino auto-contención absoluta. No obstante, la materia aún tiene un carácter derivado. No da desde sí misma la vida y la evolución.

\section{Incorporación de las leyes de la termodinámica en un universo en continua creación}

Las leyes de la termodinámica son incorporadas por Bergson en el universo abierto y en construcción, como expresión de la materia, válidas sólo en cada mundo en particular (Conry, 2000: 215). Las leyes de la termodinámica se aplican pues a sistemas aislados, a los mundos que a semejanza de nuestro sistema solar pueden considerarse aislados naturalmente.

Podemos aplicar las leyes de nuestra física a nuestro propio mundo [sistema solar], y sin duda extenderlas a cada uno de los mundos tomados aisladamente, pero nada indica que ellas se apliquen también al universo entero, ni incluso que una afirmación semejante tenga algún sentido, pues el universo no está hecho, sino que se hace sin cesar. Crece sin duda indefinidamente por la adjunción de mundos nuevos (Bergson, 2007: 248). 
En la concepción local de la termodinámica, Bergson sigue a Boltzmann ${ }^{1}$ y su concepción local de la segunda ley, pero el universo esbozado aquí es diametralmente opuesto. Es un universo vivo, abierto, de noequilibrio, en continua construcción, y no un universo muerto, en equilibrio y estabilidad eterna. Expresado aquí por la imagen del vapor lanzado al aire en una caldera con fisuras ${ }^{2}$.

Imaginemos pues un recipiente lleno de vapor a una tensión elevada, y aquí y allá, en las paredes del mismo, una fisura por donde el vapor se escapa a chorros. El vapor lanzado en el aire se condensa casi por entero en gotitas que vuelven a caer, y esta condensación y esta caída representan simplemente la pérdida de algo, una interrupción, un déficit. Pero una pequeña parte del chorro de vapor subsiste, no condensado, durante algunos instantes; esta parte se esfuerza por elevar las gotas que caen; llega, a lo sumo, a hacer más lenta su caída. Del mismo modo, de un inmenso reservorio de vida deben brotar chorros sin cesar, cada uno de los cuales, al caer, es un mundo. La evolución de las especies vivientes al interior de ese mundo representa lo que subsiste de la dirección primitiva del chorro original y de un impulso que se continúa en sentido inverso de la materialidad (Bergson, 2007: 253).

A partir del impulso vital se constituyen por el movimiento inverso mundos o sistemas solares como el nuestro, donde son válidas las leyes de la termodinámica. Cada uno de estos mundos está en una caída constante, disipando energía hacia la muerte térmica. "Un mundo como nuestro sistema solar aparece como agotando a todo instante algo de la mutabilidad que contiene" (Bergson, 2007: 250). Cada mundo tiende a un equilibrio final, hacia una homogeneidad total, hacia la espacialidad geométrica (partes extra partes). Los seres vivos y su evolución retardan la caída de la materia, la degradación de la energía, pero no pueden evitarla (Bergson, 2007: 252). La «porción» de vitalidad en cada mundo es finita (Bergson, 2007: 260). El impulso infinito, el élan vital, sigue su curso, se crean nuevos mundos, mientras que los ya creados se van adormeciendo en la materialidad. El impulso vital constituye la energía espiritual (usando una expresión del propio Bergson), el principio extra-espacial que

1 Bergson cita las Lecciones de Boltzmann en estos pasajes de La evolución creadora (Bergson, 2007: 251).

2 No hay que tomar, sin embargo, demasiado al pie de la letra estas metáforas o imágenes, como el mismo Bergson nos advierte (Bergson, 2007: 254), en este caso porque se trata de una imagen puramente material que deja de lado la libertad e indeterminación propias de lo vivo. Ninguna imagen puramente material nos dará una idea plena de la vida.

VERITAS, No 34 (Marzo 2016) 
permitiría mantener el continuo cambio del universo, su "mutabilidad" (Bergson, 2007: 251). El élan vital sería la fuente de la energía que se degrada. "Todos nuestros análisis nos muestran en la vida un esfuerzo por remontar la pendiente que la materia desciende" (Bergson, 2007: 252). Sólo cobra sentido y se comprende la actividad de la naturaleza expresada por la segunda ley en el marco más general del impulso original, de este impulso que es a la vez fuente de la vida y la materia. Si hay una mutabilidad inicial, es porque hay una diferencia que la posibilita, y esta inestabilidad inicial "a la cual debemos la riqueza y la variedad de los cambios que se cumplen en nuestro sistema solar" (Bergson, 2007: 250), no es otra que el propio impulso vital (Bergson 2007: 252). Si existen nuestro mundo y sus cambios es porque no sólo hay movimiento de degradación, no sólo hay materia, sino porque en última instancia hay vida. El universo no está en equilibrio o reposo, porque existe una energía, el impulso vital, en constante renovación.

En este universo, como hemos visto, Bergson interpreta las leyes de la termodinámica como leyes locales, no universales. Al mismo tiempo, les asigna una distinta valoración en cuanto a su relevancia metafísica, es decir, en cuanto a su mayor o menor capacidad para expresar el dinamismo del élan vital, la durée (duración) o tiempo real. La primera ley de la termodinámica, extiende, para Bergson, las leyes de conservación de la mecánica, pues implica reversibilidad y por tanto anulación del tiempo (Bergson, 2007: 248) ${ }^{3}$. La primera ley establece la equivalencia de todas las transformaciones, por tanto es una ley propia de un mundo de reversibilidades, donde el tiempo no juega ningún rol. La propia noción de energía implica una noción estática, como ya hemos esbozado al hablar de Thomson, quien se refiere a la energía "almacenada". Sin embargo, Bergson destaca un aspecto positivo de la primera ley (Bergson, 2007: 249) ${ }^{4}$. Desde una perspectiva metafísica, más allá de su carácter puramente cuantitativo de "permanencia objetiva de cierta cantidad de cierta cosa" (Bergson, 2007, 249), indicaría la relación de un fragmento del mundo respecto a otro, afirmaría la relación mutua de las distintas

3 Las leyes de conservación de la mecánica, o la primera ley de la termodinámica que es una extensión de ellas, implican reversibilidad y por tanto anulación del tiempo. Rige aquí un tiempo espacializado, un tiempo como línea espacial, que es indiferente al proceso que mide, que impone su medida a cada proceso sin tomar en cuenta la naturaleza particular de cada uno de ellos, cf. (Bergson, 2007: 28); también (Meyerson, 1908: 194). Bergson realiza una crítica de esta concepción del tiempo desde su primera obra (Bergson, 2006: 61).

4 Bergson cita a Pierre Duhem y su L'evolution de la mécanique, de donde probablemente toma esta concepción metafísica de la primera ley. 
partes de cada sistema aislado, "la necesidad para todo cambio que se produce de ser contra-balanceado, en alguna parte, por un cambio de sentido contrario" (Bergson, 2007: 249). Por su parte, la segunda ley, ley de degradación de la energía, nos señala, según Bergson, "sin símbolos interpuestos, sin artificios de medida, la dirección en la que marcha el mundo" (Bergson, 2007: 250) (al menos la dirección en que marcha la materia). Es por esto para Bergson "la más metafísica de las leyes de la física" (Bergson, 2007: 250), no meramente una ley cuantitativa, como la ley de conservación de la energía, sino que expresión de algo más general que no es solamente formulable en términos matemáticos. Es una ley metafísica y no puramente matemática porque expresa algo absoluto, un sumergimiento en la duración misma (Meyerson, 1908: 262). La segunda ley o principio de Carnot-Clausius, implicaría una novedad radical en la física, al introducir la temporalidad, la irreversibilidad. El principio de Carnot "reintegra en la ciencia las consideraciones del tiempo real, en la misma medida que los principios de primer género [conservación] implican la eliminación del tiempo" (Bergson, 2011: 369). La segunda ley expresa esencialmente la duración misma, el carácter irreversible de esta duración, pero en el sentido opuesto, el sentido de la materia.

\section{Unidad de vida y materia en los seres vivos y la evolución crea- dora}

En el universo dibujado por Bergson, los seres vivos poseen un carácter especial, en ellos aprehendemos la doble tendencia de vida y materia. Los seres vivos son análogos al universo en su conjunto, son sistemas abiertos, de no-equilibrio, en continua creación, con límites naturalmente impuestos y no artificiosamente construidos por nuestra inteligencia orientada siempre hacia la acción (Bergson, 2007: 34). La individualidad de un ser vivo no implica cierre absoluto, como el aislamiento termodinámico, sino cierre y apertura a la vez (Bergson, 2007: 32). Los seres vivos son unión de vida y materia. En su organización se dan la mano vida y disipación, el movimiento "ascendente" y "descendente" de la naturaleza, constituyendo una suerte de modus vivendi, una suerte de acuerdo entre vitalidad y materialidad.

En realidad la vida es un movimiento, la materialidad es el movimiento inverso, y cada uno de esos dos movimientos es simple, siendo la materia que forma un mundo un flujo indiviso, siendo también indivisa la vida que la atraviesa recortando en la materia seres vivientes. De esas dos corrientes, la segunda es contraria a la primera, pero la primera obtiene no obstante 
algo de la segunda: de allí resulta un modus vivendi entre ellas, que es precisamente la organización (Bergson, 2007: 256).

La organización en que consiste un ser vivo es unidad de impulso y agregación de partes a la vez (Bergson, 2007: 107). El ser vivo es unidad de impulso y disipación, es organización disipativa. En la organización en que consiste el ser vivo se dan la mano creación y disipación.

La actividad vital consiste esencialmente en acumulación de energía y ocupación explosiva de ella (Bergson, 2007: 261). De esta manera, la vida retarda la "caída" de la materia, la degradación de la energía, e introduce en la materia indeterminación y libertad (Bergson, 2007: 269). Indeterminación que indica elección (Bergson, 2007: 267). La elección como característica de la vida no implica una visualización de un futuro o respuesta preexistente, sino la posibilidad de articular distintas respuestas o tendencias frente a un estímulo dado. Entre más libre es el ser vivo, más libre es su respuesta frente a una circunstancia. No es mero automatismo. Una misma circunstancia puede dar lugar a creaciones y comportamientos diversos (Bergson, 2007: 191).

La tensión de vida y materia, de libertad y necesidad, de acumulación de energía y ocupación explosiva, de elección, no constituye solamente el mecanismo de la organización vital, sino también de la evolución biológica (Bergson, 2007: 259). La evolución creadora muestra las distintas maneras en que esta actividad esencial se ha ido configurando, muestra las distintas elecciones (Bergson, 2007: 260). Las virtualidades del impulso vital, confusamente entremezcladas, sin forma concreta o particularidad, adquieren una individualidad en el curso de la evolución gracias al movimiento inverso o de cierre de la materia: "No hay más que una cierta corriente de existencia y la corriente antagónica; de allí toda la evolución de la vida" (Bergson, 2007: 194). El impulso vital se bifurca en distintas ramas evolutivas en su encuentro con la materia (Bergson, 2007: 260). La serie de los seres vivos y los diversos caminos emprendidos por la evolución y en los que consiste la evolución poseen un carácter contingente, aunque no completamente arbitrario. Son concreciones de las virtualidades del impulso, las tendencias confusas que lleva en sí, y la clausura operada por la materia, la individuación de la materia. "Es visible que el esfuerzo ha encontrado resistencias en la materia que utilizaba; ha debido bifurcarse en su camino, repartir entre líneas de evolución diferentes las tendencias de que estaba lleno; se ha desviado, ha retrocedido; a veces, incluso se ha detenido definitivamente" (Bergson, 1963: 852). La evolución es unidad de impulso y diversidad de caminos, al igual que cada vida particular es unidad de impulso y diversidad de partes. El impulso se extiende en la evolución a través de la herencia. "La herencia 
no transmite solamente los caracteres; ella también transmite el impulso en virtud del cual los caracteres se modifican, y ese impulso es la vitalidad misma" (Bergson, 2007: 239, 45) ${ }^{5}$.

En esta imagen de la naturaleza, la vida y su evolución se oponen y se conjugan con la materia, y sólo son posibles gracias a este doble movimiento de impulso y oposición. El universo como un todo está abierto y en constante creación, en constante des-equilibrio. La irreversibilidad y la imprevisibilidad son el signo manifiesto de esta construcción en curso. En este universo vivo Bergson incorpora las leyes de la termodinámica, como expresión del carácter propio de la materia. Válidas en sistemas aislados como nuestro sistema solar, indican el camino hacia el equilibrio, hacia la espacialidad como tendencia propia del dinamismo de la materia. Esta materia es al mismo tiempo ingrediente fundamental en la configuración de la vida. Sin ella no es posible la organización de los seres vivos. Bergson aúna así disipación y vida. La actividad disipativa, los procesos irreversibles, no indican solamente un camino de degradación y homogeneidad, no sólo una pérdida, sino a la vez una oportunidad.

La verdad es que la vida es posible en todos los lugares en los que la energía descienda la pendiente indicada por la ley de Carnot y en los que una causa, de dirección inversa, pueda retardar el descenso, es decir, sin duda, en todos los mundos que penden de todas las estrellas (Bergson, 2007: 262).

Donde hay disipación hay energía disponible. Donde hay disipación es posible la vida. La vida y su evolución continúan el impulso original, pero sólo pueden ser tales en un universo material, en mundos que "caen" y en esa caída cierran y dan cuerpo al propio impulso, haciéndolo ir más allá de sí mismo.

\section{Conclusiones: mantenimiento de la dualidad vida y materia en Bergson}

Con su propuesta de un universo creativo y vital, Bergson intenta dar solución a dos cuestiones clave planteadas por la termodinámica clásica: en primer lugar, la oposición radical entre vida y materia en un universo disipativo, que de manera continua e irreversible se dirige a un estado de reposo o equilibrio, y que vuelve inexplicable y extrínseca la actividad vital y su evolución creativa; y en segundo lugar, la relación

5 Bergson toma aquí la idea de "la continuidad del plasma germinativo" de Weismann (Bergson, 2007: 45).

VERITAS, No 34 (Marzo 2016) 
existente entre las propias leyes de la termodinámica y la evolución biológica consideradas como visiones globales del universo o de la naturaleza.

La propuesta de Bergson intentará resolver estas dos cuestiones a la vez. Operando una suerte de inversión de lo planteado por la termodinámica clásica, incorpora la materia y las leyes termodinámicas a ella asociadas en un universo vital en continua construcción. La cuestión no es ya cómo es posible la vida en una materia que tiende al reposo, sino cómo es posible el reposo en una naturaleza en continua actividad. En este universo vivo y en continua construcción reubica las leyes de la termodinámica. Éstas no serán ya leyes universales, válidas para el universo en su conjunto, sino sólo leyes locales, propias de zonas relativamente cerradas, mundos como nuestra galaxia. De esta manera, el reposo y el equilibrio anunciados como destino inflexible por la segunda ley de la termodinámica aparecen sólo como un destino local que no impide la continua creación de mundos y formas de vida siempre renovadas. Bergson no duda de la validez de las leyes de la termodinámica, las acepta como caracterización de la naturaleza propia de la materia. La relocalización de estas leyes no implica su rechazo. Por el contrario, Bergson considera que su propuesta les otorga un fundamento metafísico más adecuado. $\mathrm{Al}$ fundarlas en un élan vital resuelve el problema del agotamiento de la energía mecánica y el equilibrio como destino universal.

Bergson intenta superar la oposición entre vida y materia, planteando su complementariedad. El élan vital, no sólo es fundamento de la actividad vital sino de la propia actividad material. En la imagen de naturaleza dibujada por Bergson, vida y materia aparecen como dos principios complementarios, necesarios ambos en la constitución de un devenir universal, en la continua creación de formas siempre nuevas. La naturaleza se articula como unidad y diversidad, como unidad de impulso, y diversidad de bifurcaciones o vías evolutivas en la evolución creadora, o unidad de impulso y diversidad de partes en la organización disipativa de los seres vivos. Esta complementariedad, sin embargo, no pone la vida y la materia al mismo nivel, sino que se mantiene la primacía de lo vital por sobre lo material, y la relación intrínseca entre ambos no resulta simétrica. La vida, en cuanto impulso vital, aparece como fundamento del dinamismo material, mientras que este dinamismo no fundamenta el dinamismo vital. La materia sólo juega un rol de cierre, un rol limitador de virtualidades propias del impulso vital. En esta perspectiva, la materia y su dinamismo, caracterizado por las leyes de la termodinámica no juegan, en sentido estricto, un rol constitutivo o creativo en la aparición de los seres vivos y en su evolución. La vida no brota de la materia, la materia no da de sí la vida. 
Se mantiene así una dualidad de principios, que no se resuelve en una unidad última ${ }^{6}$. La materia adquiere un rol protagónico en el devenir vital, pero siempre supeditada a la vitalidad. Bergson plantea una imagen dualista de la naturaleza, en la que aparecen dos principios, necesarios para la creación concreta pero radicalmente diversos. La materia se supedita a la vitalidad, y es dinámica sólo debido a ella. Existe una dualidad jerárquica que se complementa. Esta dualidad impide explicar por ejemplo la posibilidad del surgimiento de la vida a partir de la materia. Aunque a diferencia de la termodinámica clásica, en el universo de Bergson, la vida concreta deja de ser excepcional y precaria, y pasa a poblar todos los mundos de un universo abierto y en construcción, sigue sin explicarse la posibilidad de su surgimiento a partir de la materia. La apelación a un élan vital aparece como un mero postulado metafísico que no logra constituir una verdadera unidad de vida y materia, sino sólo una frágil complementariedad, una unidad abstracta sostenida de manera extrínseca. Para autores como Prigogine, el problema fundamental yacería en que las leyes de la termodinámica clásica al ser leyes de sistemas aislados o cerrados, de sistemas de equilibrio, no pueden dar cuenta del carácter abierto, y de no-equilibrio de los seres vivos. Sería necesario reformular y extender las propias leyes de la termodinámica ${ }^{7}$. Bergson reconoce la insuficiencia de las leyes de la termodinámica y las relocaliza en un universo vivo y creativo, quitándoles su carácter universal, pero las sigue considerando como caracterización adecuada de la materia. Al aceptar las leyes

6 Para algunos autores, podríamos encontrar en Bergson una especie de unidad última que superaría esta dualidad de principios (vida y materia). Esta unidad sería Dios. Bergson, sin embargo, sólo menciona a Dios en una ocasión en La evolución creadora, y aunque dicha mención podría considerarse como apuntando a una unidad última, lo dicho no resulta suficiente, considerando que la dualidad de principios aparece expresada a lo largo de todo el libro de manera recurrente. Respecto a esta discusión, Bergson (2009: 618).

7 Prigogine en su propuesta de termodinámica de sistemas abiertos y de noequilibrio sigue la imagen de naturaleza en continuo devenir y actividad creadora dibujada en La evolución creadora de Bergson, pero no acepta la dualidad de vida y materia. La materia misma deber dar de sí la vida. "En el fondo, espero haber contribuido a realizar el programa de Bergson, ¡sin ser bergsoniano! [...] Soy bergsoniano en cuanto al fondo del pensamiento pero no estoy en absoluto de acuerdo con el cisma de su visión dualista" (Benkirane, 2006: 52). Para conocer las propuestas teóricas de Prigogine, ver Prigogine \& Stengers (1994); Nicolis \& Prigogine (1977). Para un estudio más detallado de la influencia de Bergson y su concepción del tiempo como creación en la elaboración e interpretación de la teoría termodinámica de Prigogine, ver Durán (2013).

VERITAS, No 34 (Marzo 2016) 
de la termodinámica tal como estaban planteadas en la termodinámica clásica, Bergson se ve obligado a postular un principio vital ajeno a la materia, que funde y posibilite su dinamismo y su rol en la organización de los seres vivos y la evolución biológica. Bergson incorpora las leyes de la termodinámica en un mundo vital, pero no las renueva, aceptando así el valor puramente negativo de la disipación y de la materia. La irreversibilidad termodinámica indicada por el crecimiento de la entropía sigue siendo meramente sancionadora de la tendencia al equilibrio, la materia simplemente disipa o consume la energía vital que le otorga la unidad dinámica a la naturaleza. La termodinámica ha sido incorporada a un universo vital, pero no ha sido vitalizada ella misma.

\section{REFERENCIAS}

-Benkirane, R. (2006). La complexité, vertiges et promeses. Paris: Le Pommenier.

-Bergson, H. (1963). La energía espiritual. En H. Bergson, Obras escogidas (págs. 833-1020). Madrid: Aguilar.

-Bergson, H. (1963a). Pensamiento y movimiento. En H. Bergson, Obras escogidas (págs. 1021-1284). Madrid: Aguilar.

-Bergson, H. (2006). Ensayo sobre los datos inmediatos de la conciencia. Salamanca : Sígueme.

-Bergson, H. (2007). La evolución creadora. Buenos Aires: Cactus.

-Bergson, H. (2009). L'évolution créatrice (édition critique). Paris: Presses Universitaires de France.

-Bergson, H. (2011). Écrits philosophiques. Paris: Presses Universitaires de France.

-Bertalanffy, L. v. (1933). Modern Theories of Development. London: Oxford University Press.

-Boltzmann, L. (1895). On Certain Questions of the Theory of Gases. Nature, 28, 413-415.

-Boltzmann, L. (1964). Lectures on Gas Theory. New York: Dover.

-Boltzmann, L. (1972). Über die Beziehung zwischen dem zweiten Hauptsatze des mechanischen Wärmetheorie, 1972. Consultado el 12 12, 2012, en Joël Le Roux, Prof. l'École Polytechnique de l'Université de Nice: http://users.polytech.unice.fr/ leroux/boltztrad.pdf

-Boltzmann, L. (1986). Escritos de mecánica y termodinámica. Madrid: Alianza.

-Brush, S. G. (1976). The Kind of Motion We Call Heat, Book. 2. Amsterdam: North-Holland Publishing Company.

-Carnot, S. (1987). Reflexiones sobre la potencia motriz del fuego y las máquinas adecuadas para desarrollar esta potencia. Trad. J. O. Ordóñez. Madrid: Alianza.

-Clausius, R. (1867). On Several Convenient Forms of the Fundamental Eqs. of the Mechanical Theory of Heat (9th Memoir). En R. Clausius, The Mechanical Theory of Heat (págs. 327-365). London: John Van Voorst.

-Clausius, R. (1868). On the fundamental theorem of the mechanical theory of heat. Philosophical Magazine and Journal of Science 35.Serie 4.239, 405-419. 
-Conry, Y. (2000). L'évolution créatrice d'Henri Bergson. Investigations critiques. Paris: L'Harmattan.

-Durán, R. (2013). Autoorganización y estructuras disipativas : la imagen de naturaleza de Ilya Prigogine, P. Universidad Católica de Valparaíso. Tesis inédita.

-Glansdorff, P. \& I. Prigogine. (1971). Thermodynamic Theory of Structure, Stability and Fluctuations. London: John Wiley \& Sons.

-Gunter, P. A. (2007). Bergson's Creation of the Possible. SubStance 36 (3), 3341.

-Kragh, H. (2008). Entropic Creation: Religious Contexts of Thermodynamics and Cosmology. Ashgate.

-Meyerson, E. (1908). Identité et realité. Paris: Felix Alcan.

-Needham, J. (1942). Evolution and Thermodynamics: A Paradox with Social Significance. Science \& Society, 6 (4), 352-375.

-Nicolis, G. \& I. Prigogine. (1977). Self-Organization in Nonequilibrium Systems. From Dissipative Structures to Order through Fluctuations. John Wiley \& Sons.

-Prigogine, I. \& P. Glansdorff. (1973). L'écart à l'équilibre interpreté comme une source d'ordre. Structures disipatives. Bulletin de la Classe des Sciences, Académie Royale de Belgique, 59, 672-702.

-Prigogine, I. \&. I. Stengers (1994). La nueva alianza. Madrid: Alianza.

-Thomson, W. (1882). On a Universal Tendency in Nature to the Dissipation of Mechanical Energy. En W. Thomson, Mathematical and Physical Papers, Vol. I (págs. 511-514). Cambridge: Cambridge University Press.

-Thomson, W. (1889). Popular Lectures and Addresses. London: MacMillan and Co.

-Thomson, W. (1892). The kinetic theory of the dissipation of energy. The London, Edinburgh and Dublin Philososphical Magazine and Journal of Science, 33 (5) January-June, 291-299.

Sumario: Introducción: la oposición de vida y material; 1. Termodinámica clásica: la vida en un universo que muere; 2. Bergson: vida y materia en un universo en creación continua; 3. Incorporación de las leyes de la termodinámica en un universo en continua creación; 4. Unidad de vida y materia en los seres vivos y la evolución creadora; Conclusiones: mantenimiento de la dualidad vida y materia en Bergson; Referencias. 